\title{
Strange Baryonic Resonances and Resonances Coupling to Strange Hadrons at SIS Energies
}

\author{
L. Fabbietti for the HADES Collaboration \\ e12, Physik Department Technische Universität München \\ Excellence Cluster "Origin and Structure of the Universe"
}

\begin{abstract}
The role played by baryonic resonances in the production of final states containing strangeness for proton-proton reactions at $3.5 \mathrm{GeV}$ measured by HADES is discussed by means of several very different measurements. First the associate production of $\Delta$ resonances accompanying final states with strange hadrons is presented, then the role of interferences among $\mathrm{N}^{*}$ resonances, as measured by HADES for the first time, is summarised. Last but not least the role played by heavy resonances, with a mass larger than $2 \mathrm{GeV} / \mathrm{c}^{2}$ in the production of strange and non-strange hadrons is discussed. Experimental evidence for the presence of a $\Delta(2000)^{++}$are presented and hypotheses are discussed employing the contribution of similar objects to populate the excesses measured by HADES for the $\Xi$ in $A+A$ and $p+A$ collisions and in the dilepton sector for $A+A$ collisions. This extensive set of results helps to better understand the dynamic underlaying particle production in elementary reactions and sets a more solid basis for the understanding of heavy ion collisions at the same energies and even higher as planned at the FAIR facility.
\end{abstract}

Keywords: resonances, strangeness, elementary collisions

PACS: $14.20 . \mathrm{Gk}, 25.70 . \mathrm{Ef}, 14.20 . J n, 14.40 .-\mathrm{n}, 13.75 .-\mathrm{n}, 14.20 .-\mathrm{c}, 14.40 .-\mathrm{n}$

\section{INTRODUCTION}

The role played by resonances in hadron production in elementary and heavy ions collisions in the GeV energy range turned out to be fundamental even at a kinetic energy of $3.5 \mathrm{GeV}$. Previously it has been indeed thought that around $\sqrt{s}=3 \mathrm{GeV}$ the contribution by baryonic resonances should become less important. The HADES collaboration has published in the last three years several measurements that confirm the importance of the baryonic resonances associated to or leading to strangeness production providing new information for some exclusive production channels. Hence a first aspect consists in the quantitative determination of the resonance production associated to a strange ( or non strange) final state. As a matter of fact all the models used to interpret the available data in $\mathrm{p}+\mathrm{p}, \mathrm{p}+\mathrm{A}$ and $\mathrm{A}+\mathrm{A}$ collisions do not include any interferences among the possible contributing resonances and in this work we show a clear example where the interference pattern is very well recognisable and hence not negligible. So not only resonances join the production of strange hadrons, but different resonances decaying into the same final state may interfere and the cross sections and angular distribution will be influenced by this process. This second aspect also represents one of the fundamental questions of our field at the moment. The dynamics of particle production in elementary collisions is clearly interesting on its own but it also represents an important and necessary reference to interpret the experimental data collected for heavy ion reaction, where in the $\mathrm{GeV}$ energy range matter is supposed to get highly compressed during the collisions and hence new properties of the hadrons within this compressed matter might become visible. For this reason, a third aspect of the resonance study is the sequential decay of different resonances starting with rather massive objects and their production probability in elementary and heavy ion collisions. The excitation of heavy resonances with respect to the available phase space for a given reaction has been not considered up to now in the interpretation of the data for $\mathrm{p}+\mathrm{p}, \mathrm{p}+\mathrm{A}$ and $\mathrm{A}+\mathrm{A}$ reactions in the $\mathrm{GeV}$ region, and aside the strangeness sector the population of these heavy resonances might also modify the measured yield of non-strange observables. The question which can be asked in this context is if at these energies the observed excesses for dilepton in the intermediate mass range or the production of the $\Xi$ in heavy ion collisions might also be linked to the decay of massive resonances. In the following these three different aspects of the resonance production results will be discussed on the base of the recent HADES. 


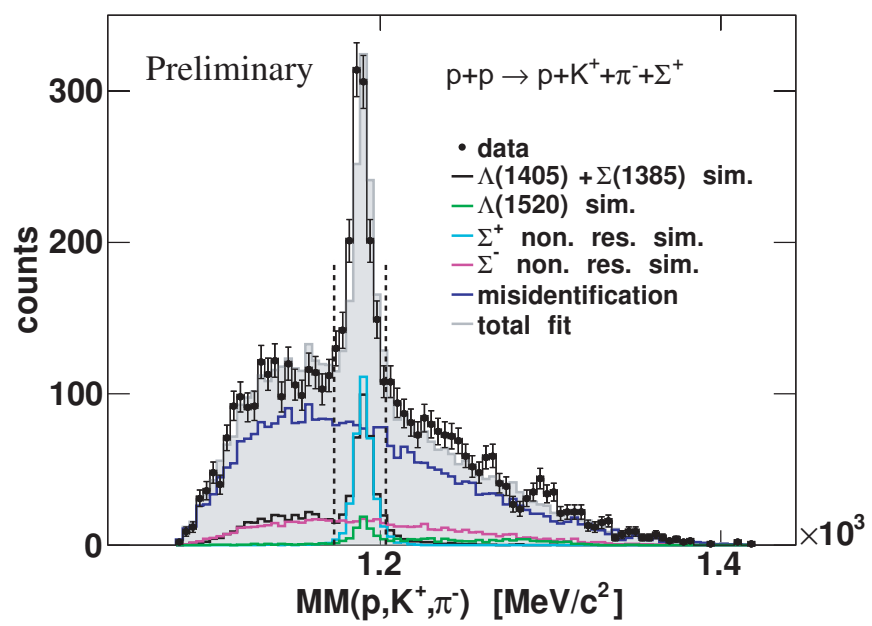

FIGURE 1. (Color online) Missing mass to $\left(p, K^{+}, \pi^{-}\right)$showing the $\Sigma^{+}$signal together with the misidentification background [1]. The vertical dashed lines represent the $3 \sigma$ cuts applied to select the $\Sigma^{+} \pi^{-}$events.

\section{ASSOCIATE PRODUCTION OF RESONANCES TO STRANGE HADRONS}

We begin with considering the investigation of the $\Lambda(1405)$ resonance produced in $\mathrm{p}+\mathrm{p}$ collisions at $3.5 \mathrm{GeV}$ $[2,3]$. Here the reaction $p+p \rightarrow \Lambda(1405)\left(\rightarrow \Sigma^{ \pm}+\pi^{\mp}\right)+p+K^{+}$is analysed considering also the successive decay $\Sigma^{ \pm} \rightarrow \pi^{ \pm}+n$. All the charged particles in the final state are identified, and the missing mass to $\mathrm{K}^{+}$, p and $\pi^{ \pm}$allows to tag the $\Sigma^{\mp}$ as it is visible in Fig. 1 [1]. The missing mass to all charged particles shows a clear signal corresponding to the neutron that can hence be selected [1]. This way, a semi-exclusive analysis could be carried out. The $\Lambda(1405)$ spectral shape is studied by means of the missing mass to the $\mathrm{K}^{+}$-p pairs. Figure 2 shows the obtained distributions where one can see the contribution by the non resonant channels $p+p \rightarrow \Sigma^{ \pm}+\pi^{\mp}+K^{+}+p$.

The data interpretation is carried out by assuming that interferences are occurring among the intermediate states and the observed shift of the $\Lambda(1405)$ spectral shape towards lower masses with respect to the nominal value has been discussed in [2,3]. The aspect of this analysis that should be underlined here is the study of the channel $p+p \rightarrow \Sigma^{-}+\pi^{+}+p+K^{+}$, non-resonant in the $\Sigma^{-} \pi^{+}$state. This contribution is clearly visible in panel b of Fig. 2 (red histogram). By looking at the $\mathrm{p} \pi^{+}$invariant mass distribution for all the selected events, the experimental data are not reproduced correctly by simulation assuming a phase-space distribution of the channel $p+p \rightarrow \Sigma^{-}+\pi^{+}+p+K^{+}$. The agreement improves consistently if the reaction $p+p \rightarrow \Sigma^{-}+\Delta(1232)^{++}+K^{+}$is considered for the total yield of the non resonant background in the $\Lambda(1405)$. The comparison between the two assumptions is shown in the left and right panel of Fig. 3 [4], where the p $\pi^{+}$invariant mass experimental distribution is compared to simulations including the phase-space background distribution (left panel) and a simulation that contains the $\Delta(1232)^{++}$resonance (right panel). It is clear that the experimental data favour the $\Delta(1232)^{++}$hypothesis.

For the charge conjugated channel $p+p \rightarrow \Sigma^{+}+\pi^{-}+p+K^{+}$no resonance is clearly visible in the p $\pi^{-}$invariant mass (see Fig. 2 in[4]). In this case the formation of one or more rather broad $\mathrm{N}^{*}$ resonances could occur but it would not be easy to detect them in the invariant mass spectrum because of their large widths. On the other hand one can not exclude that also in this case the resonance formation replaces the phase-space emission of the same final state.

A similar case is discussed in the analysis of $K_{S}^{0}$ produced in $\mathrm{p}+\mathrm{p}$ collisions at $3.5 \mathrm{GeV}$ [5], where the formation of a $\Delta^{++}(1232)$ resonance accompanying the $K_{S}^{0}$ production is strongly dominant with respect to the phase-space emission of the $\mathrm{p} \pi^{+}$pairs if the following reactions are compared:

$p+p \rightarrow \Delta^{++}+K_{S}^{0}+\Lambda / \Sigma$,

$p+p \rightarrow p+\pi^{+}+K_{S}^{0}+\Lambda / \Sigma$.

Again the $\mathrm{p} \pi^{+}$invariant mass reconstructed for events that contain additionally a $K_{S}^{0}$ and a hyperon allows to draw quantitative conclusions and extract production cross sections. Without going into the details of the exclusive analysis carried out to extract the cross section of the different production channels [5], the extracted p $\pi^{+}$invariant mass for 


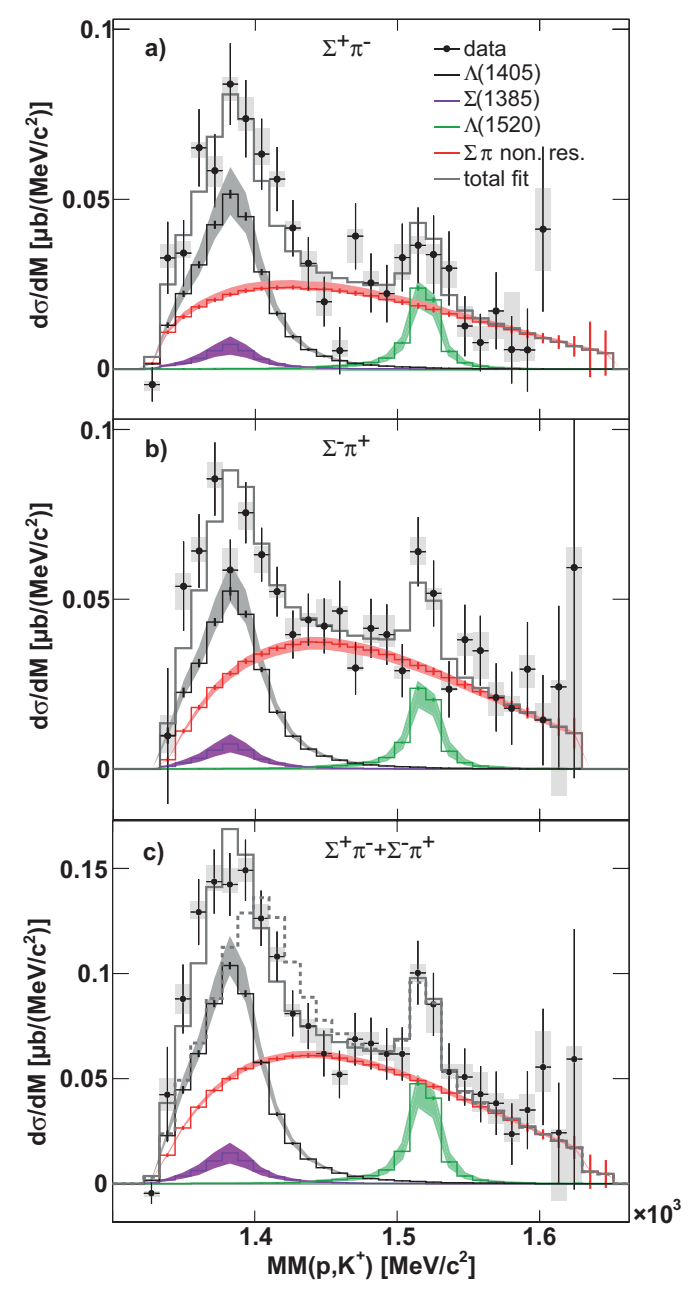

FIGURE 2. (Color online) Missing mass $M M\left(p, K^{+}\right)$distributions for events attributed to the $\Sigma^{+} \pi^{-}$decay channel a) and to the $\Sigma^{-} \pi^{+}$decay channel b). Panel c): sum of both spectra from panels a) and b) [2]. The gray dashed histogram shows the sum of all simulated channels if the $\Lambda(1405)$ is simulated with its nominal mass of $1405 \mathrm{MeV} / \mathrm{c}^{2}$. Coloured histograms in the three panels indicate the contributions of the channels (1-5) obtained from simulations. Data and simulations are acceptance and efficiency corrected. The grey boxes indicate systematic errors.

the reactions containing a $K_{S}^{0}$ together with a $\Lambda$ or $\Sigma$ hyperon are shown in Figs. 4 and 5 respectively. The experimental distributions obtained after the kaon and hyperon selections are compared to simulation including the contribution of the $\Sigma(1385)$ and the associate production of the $\Delta(1232)^{++}$summed to the experimentally determined sideband background. The blue dashed histogram in Fig. 4 and the green dashed histogram in Fig. 5 show the peak corresponding to the nominal mass of the $\Delta(1232)^{+}+$. The dashed red lines present in both Figs. represent the phase space simulation of the reaction $p+p \rightarrow \Lambda / \Sigma+p+\pi^{+}+K_{S}^{0}$ and the yield of these distributions is determined by a multi-variables fit to the experimental data [5]. It is clear from these distributions that the experimental data favour the creation of an intermediate $\Delta^{++}$resonance with respect to the phase space emission of the p $\pi^{+}$pairs; quantitatively the cross section is found to be 10 times higher. These first two examples indicate that whenever possible the resonance production dominates with respect to the phase space emission of hadrons in reactions where strange hadrons were studied. 

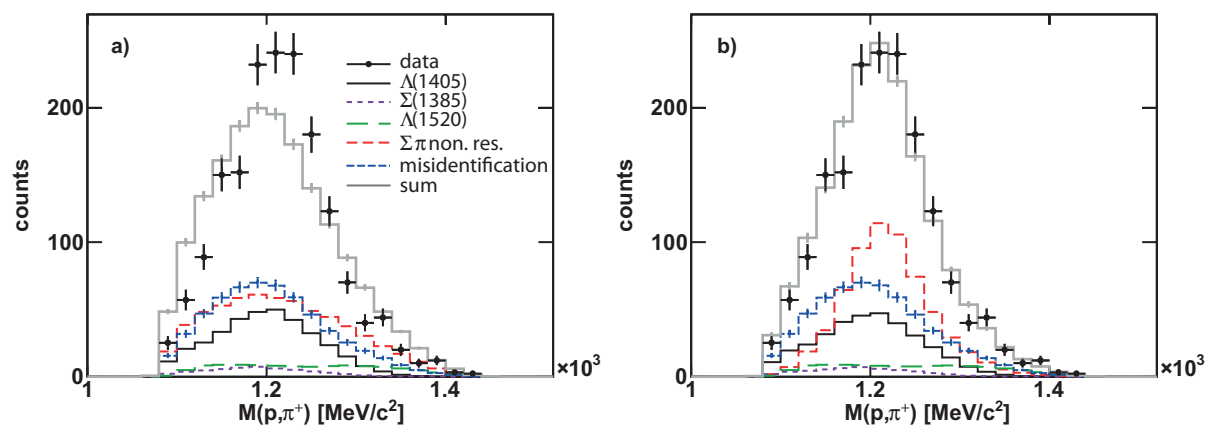

FIGURE 3. (Color online) $\mathrm{p} \pi^{+}$invariant mass distribution obtained after the $\Sigma^{-}$and $K^{+}$selection within the HADES acceptance [4]. Panel a): a phase space distribution is assumed for the $\Sigma^{-} \pi^{+}$non-resonant part. Panel b): the reaction $p+p \rightarrow \Sigma^{-}+K^{+}+$ $\Delta(1232)^{++}$is used to simulate the $\Sigma^{-} \pi^{+}$non-resonant part.

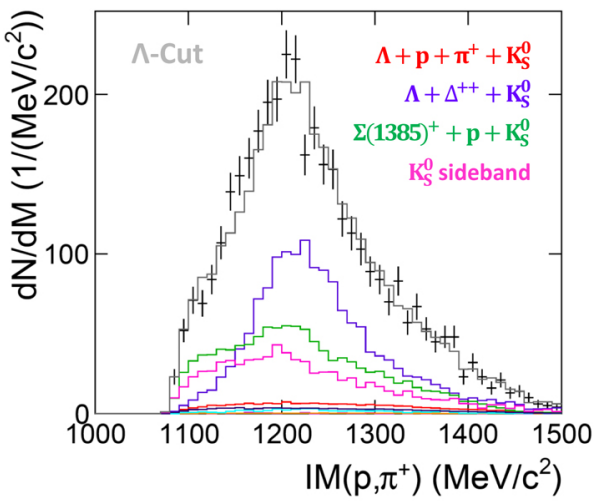

FIGURE 4. (Color online) $\mathrm{p} \pi^{+}$-invariant mass distribution after the $\Lambda$-cut on the missing mass distribution to the identified $\mathrm{p}, \pi^{+}, \pi^{+}, \pi^{-}$and with a cut on the $K_{S}^{0}$ mass in the $\pi^{+} \pi^{-}$-invariant mass spectrum [5]. The grey histogram corresponds to the sum of simulated contributions together with the background defined by the sideband sample.

\section{INTERFERENCES AMONG RESONANCES}

So far the shown analyses were carried out neglecting possible interferences among the contributing channels. As for the $\Lambda(1405)$ case, the interference scenario has been studied in [3] but it leads to unrealistic cross sections of the different channels. As for the $K_{S}^{0}$ analysis reported in [5] and discussed above, most of the investigated final states contain four or five hadrons, such that the effect of interference might not be strongly visible experimentally. On the the other hand, it has been observed by studying the reaction: $p+p \rightarrow p+K^{+}+\Lambda$ at $3.5 \mathrm{GeV}$ that not only a large fraction of the total yield is connected with the intermediate production of several $\mathrm{N}^{*}$ states $(p+p \rightarrow p+$ $N^{+}\left(1650,1710,1720,1800,1850,1900 \mathrm{MeV} / \mathrm{c}^{2}\right)$ as already discussed in [6], but also a clear signature of interference effects has been measured. The study of the interferences has been possible employing a Partial Wave Analysis (PWA) of the final states utilising the Bonn-Gatchina framework [7, 8].

Figure 6 shows the final result of the PWA analysis of the HADES data for the reaction $p+p \rightarrow p+K^{+}+\Lambda$ at $3.5 \mathrm{GeV}$ [9]. The data points within the HADES acceptance are shown together with the result of an event-by-even partial wave fit, where several $\mathrm{N}^{*}$ resonances $\left(N(1650) \frac{1}{2}^{-}, N(1710) \frac{1}{2}^{+}, N(1720) \frac{3}{2}^{+}, N(1875) \frac{3}{2}^{-}\right.$, $N(1880) \frac{1}{2}^{+}, N(1895) \frac{1}{2}^{-}$and $\left.N(1900) \frac{3}{2}^{+}\right)$and non-resonant configurations with different quantum numbers were used as hypotheses. One can see that the PWA manages to model the experimental data much better than the attempt 


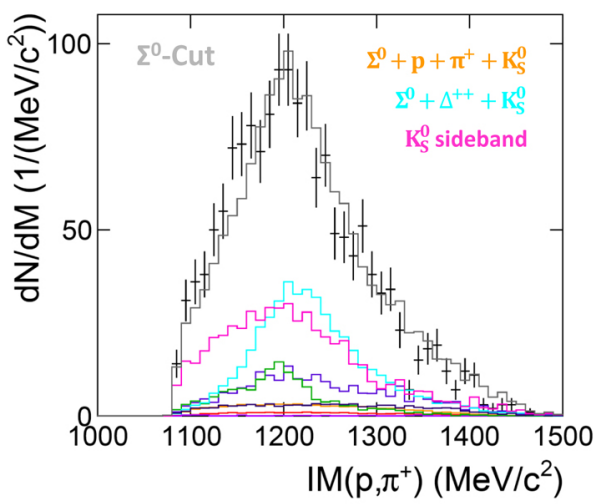

FIGURE 5. (Color online) $\mathrm{p} \pi^{+}$-invariant mass distribution after the $\Sigma$-cut on the missing mass distribution to the identified $\mathrm{p}, \pi^{+}, \pi^{+}, \pi^{-}$and with a cut on the $K_{S}^{0}$ mass in the $\pi^{+} \pi^{-}$-invariant mass spectrum [5]. The grey histogram corresponds to the sum of simulated contributions together with the background defined by the sideband sample.

shown in [10] where the incoherent sum of similar resonances has been employed. As discussed in [9] the grey band shown in Fig. 6 represents the four best solutions extracted from the PWA, which means that it has been not possible to determine the precise content of the experimental data by applying this kind of analysis to only one data set. Also, this analysis showed us that polarisation observables would help in the precise determination of the different $\mathrm{N}^{*}$ waves. For this reason, we plan to apply the PWA analysis to all the data set measured at different kinetic energies in the $\mathrm{GeV}$ energy range and available to date. This should better constraint the results and obtain an excitation function for the different $\mathrm{N}^{*}$. Several measurements of the reaction $p+p \rightarrow p+K^{+}+\Lambda$ in a fixed target configuration have been carried out by the DISTO and COSY-TOF collaborations [11] in the past and some of these data contain also polarisation observables. The available data are summarised in Table 1 together with information about the kinetic energy, statistics and availability of spin observables. One can see that the proton kinetic energy varies between 1.8 and $3.5 \mathrm{GeV}$. A global PWA of these data can constrain the excitation functions of the different $\mathrm{N}^{*}$ with a mass between 1.7 and $2 \mathrm{GeV} / \mathrm{c}^{2}$ with unprecedented precision and these data can be used as a reference of the upcoming experiments at FAIR at higher kinetic energies.

The PWA considered here interests the strange final state of $\mathrm{N}^{*}$ resonances, but analogous studies can be carried out in the one or two pions final states $\left(N^{*} \rightarrow N \pi / N \pi \pi\right)$. In [12] it is shown for the first time how for the same $\mathrm{p}+\mathrm{p}$ at $3.5 \mathrm{GeV}$ experiment the relative contribution of $\mathrm{N}^{*}$ and $\Delta$ resonances to the non-strange final states and then to the di-electron invariant mass spectrum has been determined within an extensive resonance model. This model includes appropriate angular distributions for the different contributing channels, partially extracted from exclusive measurements but it assumes an incoherent sum for all the contributing channels. In order to understand the role played by interferences in non strange final states and reconcile all the HADES analysis, a PWA has been already carried out for the non-strange final states at lower kinetic energies first [13]. There the effect of resonances manifests itself clearly but a consistent picture must still be worked out. This kind of analysis will be soon extended also to the $3.5 \mathrm{GeV}$ data.

It is clear that whatever effect the interference among resonances might have on the final spectra in $p+p$ and $n+p$ collisions, current transport calculations for such colliding systems do not include this mechanism at all. As a matter of fact for some probes, as for example the $\Lambda$ hyperons, yield and angular distribution are not correctly modelled in the transport calculations in $\mathrm{p}+\mathrm{p}$ and $\mathrm{p}+\mathrm{Nb}$ [14] collisions at $3.5 \mathrm{GeV}$. Going a step further, one can ask whether the coherence of the resonance emission is completely broken when moving from $p+p / p+n$ to $p+A$ and $A+A$ collisions or if the effect of the interference is persistent also there, at least to some extent. For the specific case of the $\Lambda$ hyperon $p_{T}$ and rapidity distributions might be washed out by scattering of the nucleons in $\mathrm{p}+\mathrm{A}$ and $\mathrm{A}+\mathrm{A}$ collisions, but since the interferences also influence cross sections, the effect could still be sizeable. Provided that A+A collisions can be seen as the superposition of multiple nucleon-nucleon collisions, interferences could play a role in the total yield of some hadrons. But assuming that hadronisation follows the creation of an intermediate state of matter [15] in $\mathrm{A}+\mathrm{A}$ collisions already in the $\mathrm{GeV}$ energy range the picture could be completely different and the effect of interferences completely negligible. It is clear that a better understanding of these processes is needed. 

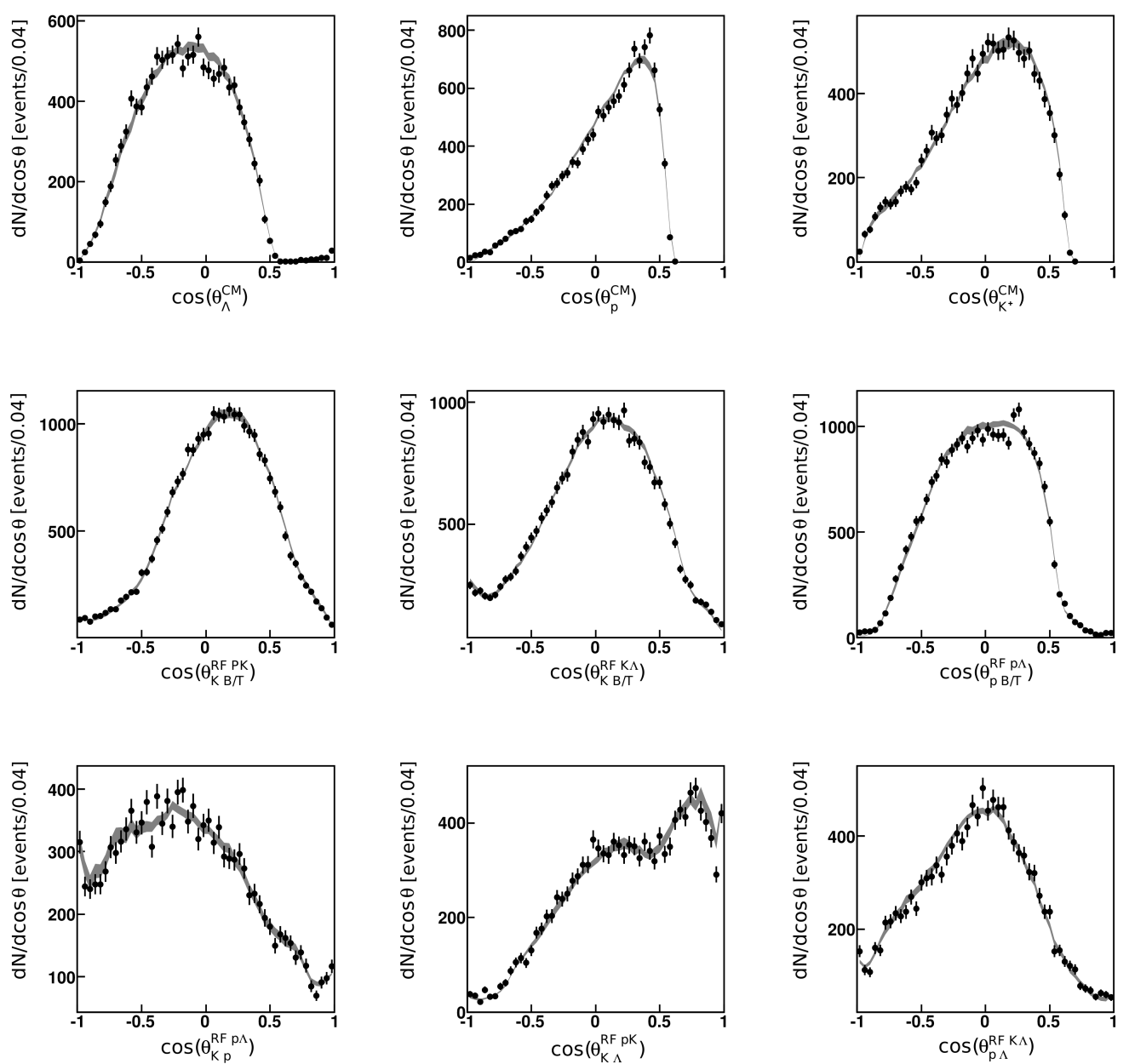

FIGURE 6. Angular correlations for the $p K^{+} \Lambda$ final state, within the detector acceptance, shown for the HADES data-set. Black dots are the experimental data with their statistical uncertainty while the grey band shows the four best solutions of the PWA and displays their systematic differences. The upper index at the angle indicates the rest frame (RF) in which the angle is displayed. The lower index names the two particles between which the angle is evaluated. CM stands for the center-of-mass system. B and T denote the beam and target vectors, respectively. The observables are: CMS angles (upper row), Gottfied-Jackson angles (middle), and helicity angles (lower row).

\section{THE CASE OF HEAVY NON-STRANGE RESONANCES}

The last but not least point of the resonance discussion in the $\mathrm{GeV}$ energy range deals with the production of heavy non-strange resonances coupling to strange final states. This story begins with the observation of the $\Sigma(1385)^{+}$in $\mathrm{p}+\mathrm{p}$ collision at $3.5 \mathrm{GeV}$ where the reaction $p+p \rightarrow \Sigma(1385)^{+}\left(\rightarrow \Lambda+\pi^{+}\right)+n+K^{+}$[16] has been investigated. This reaction has been measured semi-exclusively by detecting all the charged particles in the final state, reconstructing the intermediate $\Lambda$ hyperon via its decay into $\mathrm{p}-\pi^{-}$pairs and by applying the missing mass selection for the undetected neutron. Figure 7 shows the $\Lambda \pi^{+}$invariant mass distribution where the $\Sigma(1385)^{+}$peak is recognisable over a moderate unphysical background originating from the wrong identification of $\mathrm{K}^{+}$and a physical, non resonant background $\left(p+p \rightarrow \Lambda+\pi^{+}+n+K^{+}\right)$. The signal strength has been extracted differentially with respect to the particle emission angle by fitting the invariant mass distributions with a modified Breit-Wigner function in different bins of the angle variable. Different choices of the angular variables are possible as discussed in [16] where the differential cross sections are calculated as a function of the p-p center of mass angle, helicity and Gottfried-Jackson angles.

In order to extract a differential cross section for the $\Sigma(1385)^{+}$production acceptance and efficiency correction 


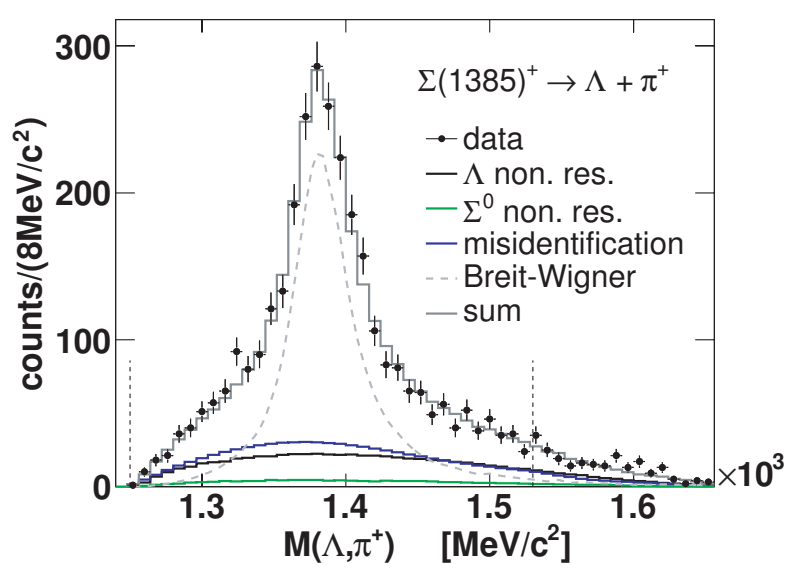

FIGURE 7. (Color online) Invariant mass distribution of the $\Lambda-\pi^{+}$pairs [16]. The circular symbols show the experimental data, the black and green curve depict the contribution by the non-resonant production, the blue curve shows the estimated misidentification background and the grey dashed curve represents a Breit-Wigner function fitted to the data. The solid grey line displays the sum of the the simulated contributions fitted to the data.

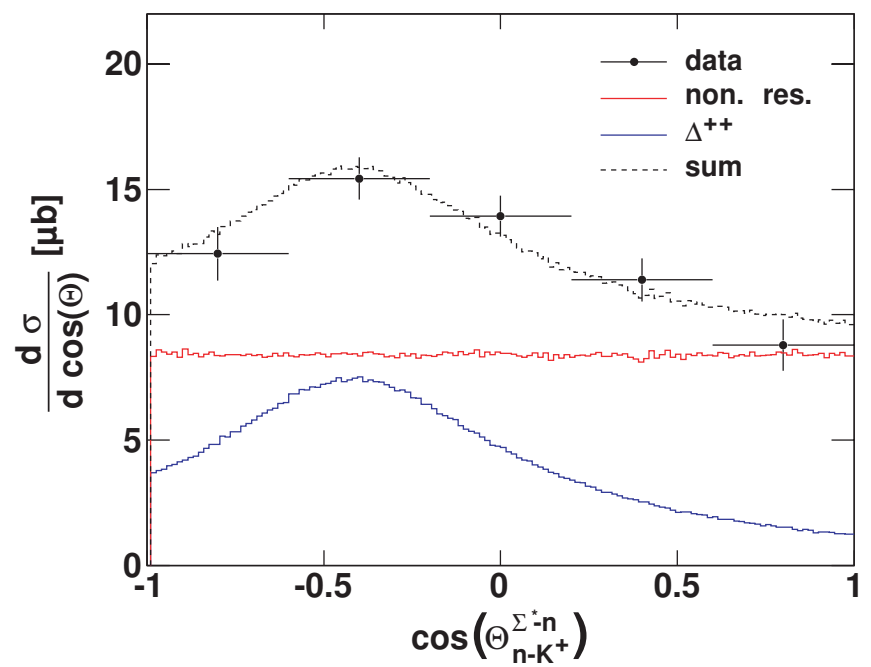

FIGURE 8. (Color online) Differential cross section of the $\Sigma(1385)^{+}$as a function of $\cos \left(\Theta_{n-K^{+}}^{\Sigma^{*}-n}\right)$ [16]. The red and blue histograms show the simulated contribution for a non-resonant production of the $\Sigma(1385)^{+}$and via an intermediate $\Delta^{++}$, respectively.

must be applied. This correction procedure requires simulations that include a model for the $\Sigma(1385)$ production, in order to extrapolate correctly the yield in the phase space region that is not covered by the acceptance of the HADES spectrometer. An iterative method is employed, as described in [1], assuming a certain production mechanism in $4 \pi$ for the $\Sigma(1385)$ and correcting the data with the simulation hypothesis. The angular distribution of the corrected data are then compared to the model and in case of deviations the model is modified and the procedure repeated.

This way, it was found that at least $30 \%$ of the $\Sigma(1385)^{+}$yield stems from the decay of a resonance with a mass of around $2000 \mathrm{MeV} / \mathrm{c}^{2}$ indicated by us as a $\Delta(2000)^{+}+$following the observation reported in [17]. The HADES data do not allow for a more exclusive analysis of the hypothetical heavy resonance nor the determination of its quantum numbers. Still, even though the available phase space for the production of a resonance with a mass larger than 2 $\mathrm{GeV} / \mathrm{c}^{2}$ is limited in $\mathrm{p}+\mathrm{p}$ reactions at $3.5 \mathrm{GeV}$, the contribution to the $\Sigma(1385)^{+}$seems relevant.

Among the most relevant results by the HADES collaboration, non-trivial in-medium effects for both strange and non strange final states have been observed in $\mathrm{Ar}+\mathrm{KCl}$ collisions at $1.756 \mathrm{AGeV}$. These results divide themselves in measured excesses of particle yields with respect to either experimental references [18] or calculation [19] and 
observed shifts of momentum distribution with respect to transport calculations [20, 21]. The deep sub-threshold production of the doubly strange hyperon $\Xi^{-}$in $\mathrm{Ar}+\mathrm{KCl}$ reactions in a fixed target configuration at kinetic energies of $1.76 \mathrm{AGeV}$ showed that the $\Xi^{-} /\left(\Lambda+\Sigma^{0}\right)$ ratio is significantly larger than the number obtained from available predictions [19] based on statistical hadronization models. Taking the NN threshold of the $\Xi$ production at $\sqrt{s}=2.5$ $\mathrm{GeV}$ it is clear that secondary collisions and eventually in-medium modification of the vacuum hadron properties could play a role here. The $\Xi^{-} /\left(\Lambda+\Sigma^{0}\right)$ ratio is found to be equal to $\left(5.6 \pm 1.2_{-1.7}^{+1.8}\right) \cdot 10^{-3}$ and more than a factor 20 larger than the value extracted from a statistical hadronization model applied to Au+Au collisions at $1.76 \mathrm{AGeV}$. One has to observe that all other hadron species measured in the $\mathrm{Ar}+\mathrm{KCl}$ experiment agree with the value predicted by the thermal model. This excess could so far not be explained, even including the contribution of reactions like $Y+Y \rightarrow \Xi+N$ in adequate transport calculation that model the heavy ion reactions [22, 23]. This measurement speaks in favour of the formation of an intermediate form of matter beyond a trivial superposition of nucleon-nucleon collisions followed by pion-nucleon and $\Delta$-nucleon secondary reactions. Indeed, in case of a deconfined phase of matter the production of double strange baryon could proceed easier in a sort of catalytic mechanism as discussed in [15].

On the other hand if one looks at recent results from p+A reaction at $3.5 \mathrm{GeV}$ [24], a similar unexpected excess for the $\Xi^{-}$production probability is found if one compares the measured value of $2.0 \pm 0.4($ stat $) \pm 0.3($ norm $) \pm$ 0.6 (syst) $) 10^{-4}$ to model prediction assuming a statistical hadronization. Provided that there is not real reason to believe that the hadronization happens governed by any thermal equilibrium in $\mathrm{p}+\mathrm{A}$ collisions in the $\mathrm{GeV}$ energy region, we still deal here with a sub threshold production for the $\Xi^{-}$.

The question can be asked whether also in this case as for the $\Sigma(1385)^{+}$production in $\mathrm{p}+\mathrm{p}$ reactions at 3.5 $\mathrm{GeV}$ the $\Xi^{-}$yield might stem from the decay of a massive strange or non-strange resonances in processes as $\Lambda(>2000) \rightarrow \Xi^{-}+K^{+}$or $\Delta^{+}(>2350) \rightarrow \Xi^{-}+K^{+}+K^{+}$. This possibility cannot be tested directly with the available data in $\mathrm{p}+\mathrm{p}$ and $\mathrm{p}+\mathrm{Nb}$ collisions exploring the same method used for the analysis of the $\Sigma(1385)^{+}$because of the reduced statistics but also because $\mathrm{p}+\mathrm{A}$ collisions do not allow for exclusive analysis. This hypothesis could be investigated in high statistics $\mathrm{p}+\mathrm{p}$ collisions with kinetic energies above the $\mathrm{N}-\mathrm{N}$ threshold of $3.7 \mathrm{GeV}$ as it will soon possible with HADES. The improved data acquisition system and particle identification capability for positive and negative kaons via the RPC detector can help to address this kind of decay via exclusive analyses in the near future.

Massive non-strange resonances could obviously also be produced in A+A collisions already at energies below the NN threshold by multiple collisions and considering the higher tails of the Fermi distribution of the momentum distribution of the single nucleon. Which means that the excess measured in $\mathrm{Ar}+\mathrm{KCl}$ for the $\Xi^{-}$baryon could also stem from the decay of an heavy resonance and not formed in a pre-hadronic state of matter. This scenario can be tested in elementary hadron-hadron collisions and then included in the modelling of heavy ion collisions to be checked against other hypotheses.

Also for the non-strange sector similar mechanisms coupled to resonances could occur. In particular, one can test hypotheses where heavy $\mathrm{N}^{*}$ resonance decay into a final state containing a $\eta$ or $\rho / \omega$ meson. An excess has been measured in the $e^{+}-e^{-}$invariant mass spectra collected for the $\mathrm{Ar}+\mathrm{KCl}$ reaction at $1.76 \mathrm{AGeV}$ with respect to an adequately normalised reference spectrum measured in $p+p$ and $p+n$ collisions at slightly lower (1.25 AGeV) kinetic energies [18].

The excess in the dilepton yield is measured in the $e^{+}-e^{-}$invariant mass region right below the $\rho$ nominal mass in correspondence of the region where the decay of $\eta$ in the dilepton final state plays and important role. These results have been interpreted by theory either advocating in-medium modification of the $\rho$ spectral shape [25] or the contribution of the decay of $\Delta$ resonances [26]. It is natural to ask to which extent the coupling of resonances to final states containing dileptons influences the invariant mass region where the excess is observed and also whether objects heavier than $1.5 \mathrm{GeV} / \mathrm{c}^{2}$ play a role here.

Exclusive measurements of the reaction $p+p->\eta / \omega+p+p$ have been carried out by measuring the two protons in the final state, employing the missing mass technique to select the $\eta$ and $\omega$ mesons and finally applying a kinematic refit to improve the momentum resolution [27]. The $\mathrm{p} \eta / \omega$ invariant mass distributions obtained after the selection of the two final states can be used to look for the signature left by the presence of resonances and in the case of the $\eta$ meson about $47 \%$ of the total yield has been attributed to the the $\mathrm{N}^{*}(1535)$ [27]. For the $\omega$ channel the data seem in agreement with a phase space production. This kind of measurements allows also to investigate whether the angular distributions of the $\eta+p+p$ and $\omega+p+p$ shows any signs of a resonance decaying into a mesonproton pair $\left(N^{*+} \rightarrow \eta / \omega+p\right)$. As discussed in the $\Sigma(1385)^{+}$analysis [16] different angles can be defined for $\mathrm{p}+\mathrm{p}$ collisions with a three body final state. As it is shown in Fig. 8 the helicity distribution with respect to $\Theta_{n-K^{+}}^{\Sigma^{*}-n}$, i. e. the angle between the $\Sigma(1385)^{+}$and the neutron in the neutron- $\mathrm{K}^{+}$reference system, shows a different pattern for 


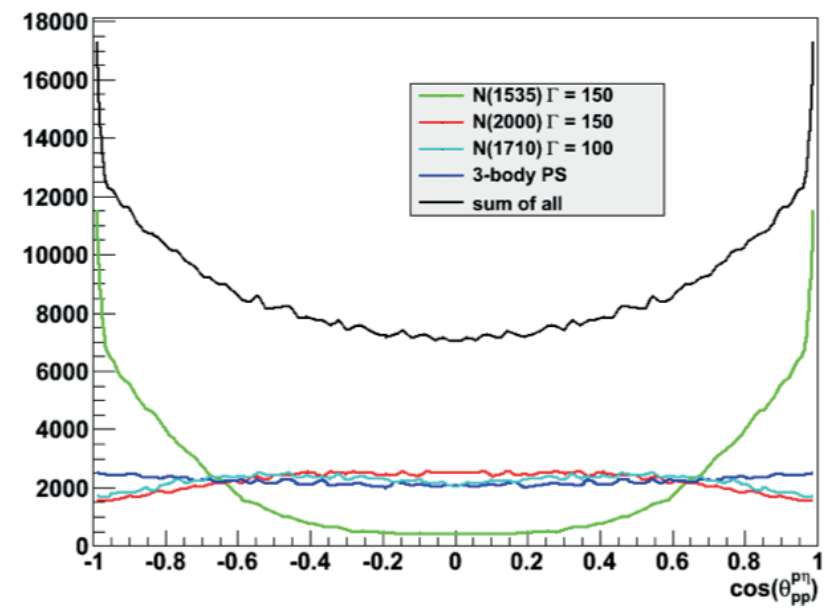

FIGURE 9. (Color online) Simulated distribution of the $\cos \left(\Theta_{p p}^{p \eta}\right)$ for the final state $p+p \rightarrow p+p+\eta$. The dark blue line represents a phase space emission, the green, red and cyan lines correspond to simulation including the intermediate production of $\mathrm{a} \mathrm{N}^{*+}$ with a mass of 1535,1710 and $2000 \mathrm{MeV} / \mathrm{c}^{2}$ respectively. The black line represents the sum of all the simulated contributions.

the non-resonant $\Sigma(1385)^{+}$emission with respect to the case $\Delta(2000)^{++} \rightarrow \Sigma(1385)^{+}+K^{+}$. The same variable can be constructed for the $\eta$ and $\omega$ missing mass analyses where then the helicity angles will be $\Theta_{p p}^{p \eta}$ and $\Theta_{p p}^{p \omega}$, angles between the $\eta / \omega$ and the proton in the final state in the reference system of the two final state protons. It is clear that since the two protons in the final state are indistinguishable, both permutations have to be considered. Figure 9 shows the results from simulations of the reaction $p+p \rightarrow p+p+\eta$ assuming either a phase space emission of the three particles in the final state or the production of an intermediate $\mathrm{N}^{*+}$ resonance with a mass of 1535,1710 and $2000 \mathrm{MeV} / \mathrm{c}^{2}$. These simulations represent the distribution in the full phase space and do not account for the detector acceptance or reconstruction efficiency and each contributions has the same weight in the total sum. It is clear from the distributions that the helicity angle measured in case of a massive resonance changes significantly with respect to the angular distribution characterising a phase space emission. In particular, a difference is also visible if one compares heavier and lighter $\mathrm{N}^{*+}$ resonances. A similar distribution as shown in Fig. 9 is obtained when simulating the reaction $p+p \rightarrow p+p+\omega$. The effeciency of the selection of the resonance contribution via the helicity angle variable should be tested after having considered the effect of the geometrical acceptance and reconstruction efficiency of the measured final state in the HADES spectrometer. Figure 10 shows the $\cos \left(\Theta_{p p}^{p \omega}\right)$ distribution for the reaction $p+p \rightarrow p+p+\omega$. The black symbols represent the experimental data reconstructed within the HADES acceptance and the green histogram shows the results of full scale simulations, which hence take into account the precise response of the HADES spectrometer, for an event generator assuming the phase space emission of the considered reaction. It is clear that the geometrical acceptance selects a region of the phase space where the different resonance-scenarios display very similar distributions in the helicity angle. In particular, one can see that the experimental data are well reproduced by the phase-space simulations for the $\omega$ channel as observed for the $\omega$-p invariant mass distribution discussed in [27].

New opportunities are offered by measuring similar final states with a pion in the initial state substituting the proton beam. Then reactions as $\pi^{-}+p \rightarrow \eta / \omega+n$ could be addressed for the tagging of different resonances. In this case, the direct detection of the $\eta$ and $\omega$ mesons becomes mandatory but the upcoming electromagnetic calorimeter within HADES [28] should help the identification of channels as $\eta \rightarrow \gamma+\gamma, \eta \rightarrow \pi^{0}+\pi^{+}+\pi^{-}$and $\omega \rightarrow \pi^{0}+\pi^{+}+\pi^{-}$. The kinematic in the case of pion beams should be less focused towards the beam direction allowing for a larger geometrical acceptance within HADES. After the successful commissioning of the pion beam at the SIS18 with the HADES spectrometer new experiments can now be planned for the near future also in this context. It is clear that the same hold for the investigations connected to the $\Xi$ production in elementary collisions and the interplay with strange and non-strange resonances. Also there pion beams can be employed to study the exclusive final states. 


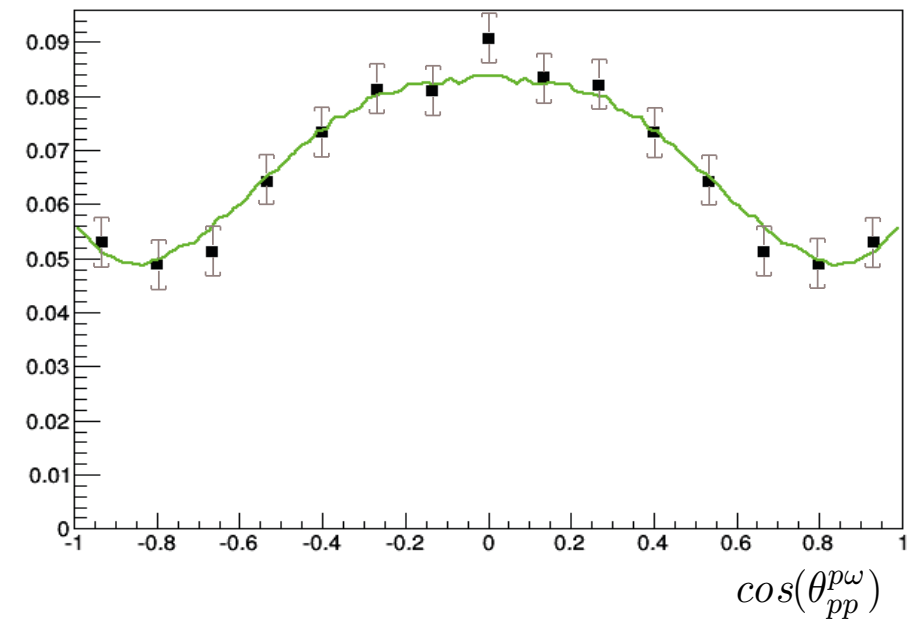

FIGURE 10. (Color online) Experimental distribution of the $\cos \left(\Theta_{p p}^{p \omega}\right)$ for the final state $p+p \rightarrow p+p+\omega$ (squared symbols) together with the distribution of the same helicity angle obtained from phase-space full scale simulations. Errors represent an estimation of the systematics.

\section{SUMMARY}

The associate production of baryonic resonances together with hyperons and kaons has been discussed and it has been shown that new exclusive analyses of the reaction $\mathrm{p}+\mathrm{p}$ at $3.5 \mathrm{GeV}$ by the HADES collaboration could pin down quantitatively the contribution of this resonances. In the specific case of the associate of the $\Delta(1232)^{++}$with $K_{S}^{0}$ a direct comparison with the phase space emission of the $\mathrm{p} \pi^{+}$pair showed that the resonant mechanism is a factor 10 more probable than the non-resonant channel. This way, the dominance of the resonances production has been verified in the studied energy regime. The second important aspect when dealing with resonances is the effect of interferences. One of the first PWA for $\mathrm{p}+\mathrm{p}$ collisions has been carried out by the HADES collaboration to describe the final state $p K^{+} \Lambda$ measured at a kinetic energy of $3.5 \mathrm{GeV}$. This analysis shows itself as suited to correctly reproduce the experimental data but presents ambiguities in the determination of the specific waves contributing to the total yield. A new enterprise is presented here, aiming to apply the same PWA published by HADES to all available data for the same reactions measured at different energies. This project will provide a more solid base for the determination of the excitation function of numerous $\mathrm{N}^{*}$ resonances in the mass range $1650-1900 \mathrm{MeB} / \mathrm{c}^{2}$. The last aspect considered in the work is the role played by heavy resonances $\left(M>2000 \mathrm{MeV} / \mathrm{c}^{2}\right)$ in the production of strange and non-strange hadrons not only in elementary but also in heavy ion collisions. It has been shown that a semi-exclusive measurement of the $\Sigma(1385)^{+}$allows to determine that about $30 \%$ of the total yield stems from the decay of a massive resonance here indicated as a $\Delta(2000)^{++}$. The possibility that a similar process could be responsible for the large $\Xi^{-}$yield in both $\mathrm{p}+\mathrm{Nb}$ and $\mathrm{Ar}+\mathrm{KCl}$ reactions is discussed, but this scenario could not be tested quantitatively yet. Similar considerations about the influence of massive objects to the production of $\eta$ and $\omega$ mesons are brought up, showing that $p+p$ collisions at $3.5 \mathrm{GeV}$ do not suit the identification of such a contribution. New perspectives offered by experiments with pion beams at GSI have been discussed as very promising.

\section{REFERENCES}

1. J. Siebenson, L. Fabbietti, A. Schmah et al. (HADES Coll.), PoS BORMIO2010:052 (2010) arxiv:1009.0946v1.

2. G. Agakishiev et al. (HADES Coll.), Phys. Rev. C87 (2013) 025201.

3. J. Siebenson and L. Fabbietti, Phys. Rev. C88 (2013) 5, 055201.

4. G. Agakichiev et al. (HADES Coll.), Nucl. Phys. A881 (2012) 178.

5. G. Agakishiev et al. (HADES Coll.), Phys. Rev. C 90 (2014) 015202.

6. S. Abd El-Samad et al. (TOF Coll.), Phys.Lett. B688 (2010) 142-149.

7. A. Anisovich, A. Sarantsev, Eur. Phys. J. A 30 (2006) 427.

8. A. Anisovich, V. Anisovich, E. Klempt, V. Nikonov, A. Sarantev, Eur. Phys. J. A 34 (2007) 129. 
9. G. Agakishiev et al. (HADES Coll.), accepted by Phys. Lett. B, arXiv:1410.8188.

10. L. Fabbietti et al. (HADES Coll.), Nucl. Phys. A914 (2013) 60-68.

11. M. Abdel-Bary et al. (COSY-TOF Coll.)) Eur. Phys. J. A 46, 27 (2010), P. Salabura et al. (DISTO Coll.)Acta Phys.Polon. B31 (2000) 2419-2429

12. G. Agakishiev et al. (HADES Coll.), Eur.Phys.J. A50 (2014) 82.

13. M.J. Amaryan et al., MesonNet 2013 International Workshop. Mini-proceedgins, arxiv:1308.2575.

14. G. Agakishiev et al. (HADES Coll.), Eur. Phys. J. A 50 (2014) 81.

15. T. Galatyuk and M. Lorenz (HADES Coll.), Central European Journal of Physics 10 (6) 1295 (2012) DOI:10.2478/s11534012-0075-2

16. G. Agakichiev et al. (HADES Coll.), Phys. Rev. C85 (2012) 035203.

17. W. Chinowsky et al., Phys. Rev. 165, 1466 (1968).

18. G. Agakishiev et al. (HADES Coll.), Phys.Rev. C84 (2011) 014902.

19. G. Agakishiev et al. (HADES Coll.), Phys. Rev. Lett. 103 (2009) 132301.

20. G. Agakishiev et al. (HADES Coll.), Phys. Rev. C 82 (2010) 044907.

21. G. Agakishiev et al. (HADES Coll.), Phys. Rev. C 90 (2014) 054906.

22. E.E. Kolomeitsev, B. Tomasik, D.N. Voskresensky, Phys.Rev. C86 (2012) 054909.

23. G. Graef, J. Steinheimer, L. Feng and M. Bleicher, arXiv:1409.7954.

24. G. Agakishiev et al. (HADES Coll.), submitted to Phys. Rev. Lett, arXiv:1501.03894.

25. J. Weil, S. Endres, H. Van Hees, M. Bleicher and U. Mosel, arXiv:1410.4206.

26. E.L. Bratkovskaya, J. Aichelin, M. Thomere, S. Vogel and M. Bleicher ,Phys.Rev. C87 (2013) 064907.

27. K. Teilab at al. (HADES Coll.), Int.J.Mod.Phys.A26 (2011) 694-696.

28. W. Czyzycki et al., Electromagnetic Calorimeter for HADES, arxiv:1109.5550. 


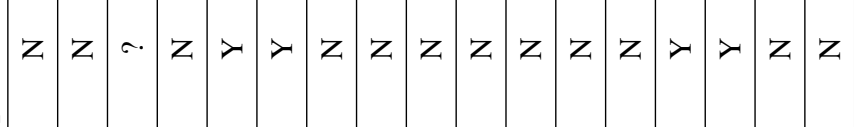
总

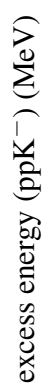

$\stackrel{\frac{0}{2}}{3}$ ปู กิ สิ

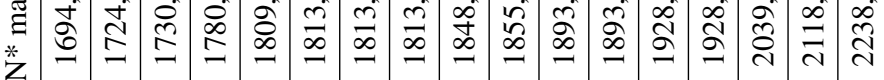
宅 $\sum_{\substack{a \\ \vdots}}^{3}$ 光

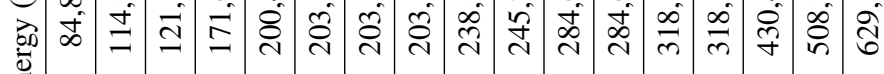
苛

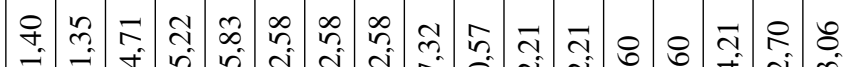

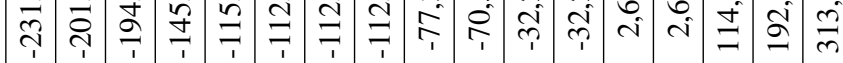

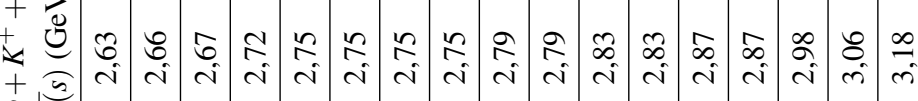
$\uparrow$

2

$+\frac{0}{2}$

莺

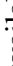

Ð

政

突

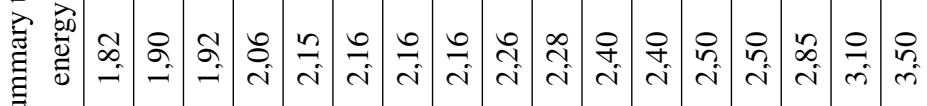

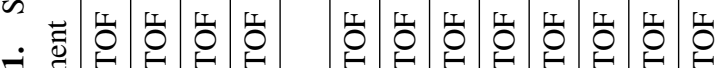

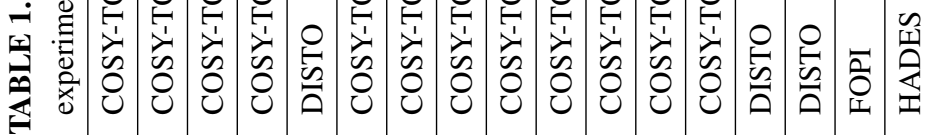

